\title{
Lennox-Gastaut Syndrome: Perspective of a Parent and a Physician
}

Heather R. McKee $\cdot$ Barbara Glasgow

Received: September 25, 2020 / Accepted: October 14, 2020 / Published online: October 28, 2020

(C) The Author(s) 2020

\section{ABSTRACT}

This article is co-authored by a parent of a 32-year-old male patient with Lennox-Gastaut syndrome (LGS) and his epileptologist. It discusses the parent's experience of having a child with LGS from diagnosis through living day-today with the disease and the physician's perspective when treating this devastating epilepsy syndrome. The patient's mother, who is his legal representative, provided written consent for publication of this article.

Keywords: Cannabidiol; Epilepsy treatment; Lennox-Gastaut syndrome; Medically refractory epilepsy; Patient perspective

\section{Key Summary Points}

Lennox-Gastaut syndrome is a devastating epilepsy syndrome which is characteristically refractory to antiseizure medications.

Despite drug resistance, antiseizure medications are the mainstay of treatment.

The study looks at the perspective of a parent and a physician regarding treatment of this syndrome.

Success with medicine can be achieved, even with refractory epilepsy syndromes, and will continue to be optimized as more focused research and targeted treatments are developed.
H. R. McKee $(\bowtie)$

Department of Neurology, Epilepsy Division, University of Cincinnati Gardner Neuroscience Institute, Cincinnati, OH, USA

e-mail: heather.mckee@uc.edu

B. Glasgow

Cincinnati, $\mathrm{OH}, \mathrm{USA}$

\section{DIGITAL FEATURES}

This article is published with digital features, including a summary slide, to facilitate understanding of the article. To view digital features for this article go to https://doi.org/10.6084/ m9.figshare.13079411. 


\section{PATIENT'S STORY FROM HIS MOTHER'S PERSPECTIVE}

I never thought I would feel relief when my son was given the diagnosis of Lennox-Gastaut syndrome. After many years of seizures, tests, medication trials and changes, and suggestions of very invasive surgeries, we finally received a definitive diagnosis that I could wrap my mind around. I knew what we were facing and we would handle it as we had handled the previous health issues my son had been dealing with since birth.

Initially we had assumed that the seizures started due to brain damage from his preterm birth. My son was 19 months old when his seizures began, and it was not until he was 10 years old that we got the diagnosis that gave us a sense of direction. We knew that, by definition, his seizures would be forever changing and very hard to control; but it is easier when you know what you are facing. For years, we kept records of each seizure as well as how often they were, how long they lasted, and what precipitated them. We did not do that any longer. It sounds weird but we were able to relax for a minute.

I never opted for surgical intervention as a form of seizure control. My son had and still has many other underlying serious medical conditions that took precedence over his seizures. Throughout his early years, he was off and on a ventilator for severe lung damage from birth. He was essentially fighting to breathe every day of his life and just to remain healthy. We did not enroll in any drug trials and, unfortunately, there were not a whole lot of new medications coming out for Lennox-Gastaut when he was young. He did receive some physical therapy for his delays over the years that would be interrupted from time to time by a seizure or an illness.

Over the years, my son has been on almost every anti-epileptic medication on the market. Some of the older medications caused adverse side effects, such as extreme drowsiness, weight loss, and agitation. Each medication was increased every 3 to 6 months with no real positive effect on seizure control for a prolonged amount of time. They all required frequent blood work to monitor the drug levels versus the effectiveness and were eventually stopped as he was started on a new medication.

As he got older, some newer medications did help with different aspects. One made his personality come out more and made him more alert with less side effects. Another controlled a different type of seizure. Another was added to stop a movement disorder that he developed in addition to his seizures. However, none of them controlled his seizures completely; yet we did not let that stop our lives. We still played, we still attended family functions, we still attempted to participate in life as much as we could, and we still do.

After 30 years of fighting this "syndrome", this thing that we could not control or predict, we have come as close as we have ever been to stopping it. To not seeing it every day. To being able to focus solely on our daily life without any interruptions. To not have to worry that even in the middle of the night a horrible seizure can come out of the blue and disrupt a good night's sleep. We have gone from almost one hundred seizures per day down to about two per month. I never thought that we would see this day. To say that two seizures per month was a good thing would have been unthinkable to us 30 years ago without the awareness brought to Lennox-Gastaut syndrome and the dedication of the researchers and neurologists from around the world. Moreover, for that... we are forever grateful.

\section{PHYSICIAN'S PERSPECTIVE}

Lennox-Gastaut syndrome (LGS) is a form of encephalopathic generalized epilepsy (EGE) and is a devastating epilepsy syndrome that typically begins within the second and sixth year of life. It is characteristically refractory to antiseizure drugs (ASDs). The diagnostic clinical triad of LGS is (1) multiple mixed seizure types, including tonic, atonic, and atypical absence with high seizure frequency, and often with status epilepticus, (2) impaired intellectual function or behavior disturbance, (3) abnormal EEG background with diffuse slow activity and characteristic epileptiform discharges described 
as slow spike-and-wave discharges while awake [1]. LGS accounts for approximately $1-4 \%$ of all childhood epilepsies [2, 3]. EGE represents approximately $11.6 \%$ of all childhood epilepsies [4].

Treatment for LGS includes efforts to manage the underlying cause of their associated cognitive and behavioral dysfunction, attempting to control seizures, and providing support for the patient's family or caretaker [1]. Despite characteristic drug resistance, ASD therapy is the primary treatment. It is a challenge to optimize seizure control and medication side effects, while maintaining a favorable quality of life. The multiple seizure types in LGS often require broad-spectrum ASDs and combination therapies. Certain medications have proven higher utility in LGS, including valproic acid, lamotrigine, and topiramate [5-7]. Felbamate is effective in seizure reduction and neurocognitive profile but is limited by serious side effects of hepatic failure and aplastic anemia [8]. Clobazam and rufinamide are also approved for LGS, and other ASDs have been reported to have possible benefit but without consistent efficacy, such as levetiracetam, zonisamide, and clonazepam [1, 9, 10]. Oral purified cannabidiol (Epidiolex), the natural cannabidiol from the marijuana plant, is an oral solution that was approved by the US Food and Drug Administration (FDA) for use in LGS in June 2018 and has shown significant effectiveness in clinical trials for refractory seizures [11-14] and for improvement in global functioning of these patients [15-18]. This was also the case for my patient.

My patient has static encephalopathy and spastic quadriplegia with diffuse cerebral volume loss. He was born by cesarean section at 28 weeks and was ventilator dependent. He has been severely delayed since birth, which worsened when his seizures started at 19 months old. His seizure history includes at least four seizure types: type 1 , staring blankly then smiles and may drool; type 2, tonic; type 3 , generalized tonic-clonic (GTC); type 4, atonic with fall. He had been on lamotrigine and levetiracetam for many years with effectiveness but continued to have daily to weekly seizures with frequent seizure clusters. His current doses are lamotrigine
$150 \mathrm{mg}$ three times daily and levetiracetam $1000 \mathrm{mg}$ three times daily and he could not tolerate higher doses. Past medication included phenobarbital, valproic acid, phenytoin, carbamazepine, and topiramate, all of which did not produce significant seizure control or caused side effects.

His mother reports him having a decline in functioning at around 10 years of age. Prior to that he was pulling up and was taking a bottle himself, but after the decline he never walked or talked; a feeding tube was placed for nutritional supplementation and a tracheostomy was placed as a result of repeated aspiration. At that time, he was initiated on primidone for abnormal movements and had remained on a dose of $100 \mathrm{mg}$ three times daily. It was reported that his seizure frequency on lamotrigine, levetiracetam, and primidone was daily of type 1 and weekly seizures of type 2,3 , and 4 . He was initiated on rufinamide in the fall of 2010 and titrated according to response to $600 \mathrm{mg}$ twice daily. As a result, his atonic seizures (type 4) resolved completely. Higher doses of rufinamide resulted in increased seizures. A vagus nerve stimulator was considered but felt to be too risky with his respiratory status. There was a lapse in care for several years and he returned to the epilepsy specialist clinic in 2018 following the approval of cannabidiol. At that point, he initially tried clobazam, but had sedation and no reduction in seizures. He was initiated on orally administered cannabidiol in January 2019. Within the first month of treatment on $2.5 \mathrm{mg} / \mathrm{kg} /$ dose twice daily his type 1 seizures had resolved, type 2 seizures were reported to be less severe and shorter in duration, and there were no type 3 seizures. His mother reported that he was more awake and alert and was doing more activity. She reported that he was "like he used to be". He started sleeping through the night and holding himself up better. It also improved his bowel motility. Since that time, his response to titration of cannabidiol has continued to reduce his seizure frequency and improve his functioning. His primary seizure that has remained refractory is type 3 , his GTC. His main precipitant is having a bowel movement and stress. These seizures continue to occur approximately two times per month and 
dosing titration has and will continue, to obtain maximal benefit.

When deciding on medication therapy for this patient, several issues were considered. First and foremost, it is critical to understand and confirm the diagnosis. For this patient, it is unclear why the diagnosis was not confirmed until he was 10 years old, but it seems that this timeframe related to his significant functional decline. Secondly, patient characteristics must be considered to tailor a medication choice. Medications can carry risks to organ systems and to quality of life. For my patient, his mother reports that it took years to make a diagnosis of Lennox-Gastaut and, therefore, treatments tried prior to this had the risk of being suboptimal. Additionally, with LGS the treatment options available are generally ineffective and refractory. As his mother stated, he tried many antiseizure medications and yet remained with excessive seizures. Over the years, certain treatments were found that were both more effective and more tolerable for this patient. With the approval of orally administered cannabidiol, the patient has been able to add its unique mechanism of action to his medication regimen and his seizure frequency has reduced substantially, down to approximately two per month.

To get my patient to a seizure frequency where he is today has not been an easy feat. It often takes trial and error of medications and dosing to obtain success with medications and this is particularly true for devastating epilepsy syndromes. Effective communication with the patient's response and the caretaker's input is invaluable to obtain an optimal treatment regimen for a particular patient. I am thankful that my patient has achieved a significant reduction in seizures from baseline and that his care can focus on other aspects of his health. Success with medicine can be achieved, even with refractory epilepsy syndromes, and will continue to be optimized as more focused research and targeted treatments are developed. I look forward to the opportunity to see this progress unfold.

\section{ACKNOWLEDGEMENTS}

Thank you to the participants of this study, both the patient and his mother.

Funding. No funding or sponsorship was received for this study or publication of this article.

Authorship. All named authors meet the International Committee of Medical Journal Editors (ICMJE) criteria for authorship for this article, take responsibility for the integrity of the work as a whole, and have given their approval for this version to be published.

Disclosures. The patient, his mother Barbara Glasgow, and Dr. Heather Ravvin McKee have nothing to disclose.

Compliance with Ethics Guidelines. The patient's mother, who is his legal representative, provided written consent for publication of this article. This article does not contain any new studies with human or animal subjects performed by any of the authors.

Open Access. This article is licensed under a Creative Commons Attribution-NonCommercial 4.0 International License, which permits any non-commercial use, sharing, adaptation, distribution and reproduction in any medium or format, as long as you give appropriate credit to the original author(s) and the source, provide a link to the Creative Commons licence, and indicate if changes were made. The images or other third party material in this article are included in the article's Creative Commons licence, unless indicated otherwise in a credit line to the material. If material is not included in the article's Creative Commons licence and your intended use is not permitted by statutory regulation or exceeds the permitted use, you will need to obtain permission directly from the copyright holder. To view a copy of this licence, visit http://creativecommons.org/licenses/by$\mathrm{nc} / 4.0 /$. 


\section{REFERENCES}

1. Wyllie E. Wyllie's treatment of epilepsy principles and practice. 6 th ed. Philadelphia: Wolters Kluwer; 2015. p. 272-83.

2. Markand ON. Lennox-Gastaut syndrome (childhood epileptic encephalopathy). J Clin Neurophsiol. 2003;20:426-41.

3. Trevatham E, Murphy CC, Yeargin-Allsopp M. Prevalence and descriptive epidemiology of Lennox-Gastaut syndrome among Atlanta children. Epilepsia. 1997;38:1283-8.

4. Camfield P, Camfield C. Long term prognosis for symptomatic (secondarily) generalized epilepsies: a population based study. Epilepsia. 2007;48: 1128-32.

5. Arzimanoglou A, Guerrini R, Aicardi J. Lennox-Gastaut syndrome in Aicardi's epilepsy in children. 3rd ed. Philadelphia: Lippincott Williams \& Wilkins; 2004. p. 38-50.

6. Motte J, Trevathan E, Arvidsson JFV, The Lamictal Study Group. Lamotrigine for generalized seizures associated with Lennox Gastaut syndrome. N Engl J Med. 1997;337:1807-12.

7. Sachedo RC, Glauser TA, Ritter F, et al. A doubleblind, randomized trial of topiramate in Lennox-Gastaut syndrome. Neurology. 1999;52: 1882-7.

8. Pellock JM, Fought E, Leppik IE, et al. Felbamate: consensus of current clinical experience. Epilepsy Res. 2006;71:89-101.

9. Ng YT, Conry JA, Drummond R, et al. Randomized phase III study results of clobazam in Lennox-Gastaut syndrome. Neurology. 2011;77: 1473-81.
10. Glauser T, Kluger G, Sachdeo R, et al. Rufinamide for generalized seizures with Lennox-Gastaut syndrome. Neurology. 2008;70:1950-8.

11. Devinsky O, Marsh E, Friedman D, et al. Cannabidiol in patients with treatment-resistant epilepsy: an open-label interventional trial. Lancet Neurol. 2016;15(3):270-8.

12. Devinsky O, Cross JH, Laux L, et al. Trial of cannabidiol for drug-resistant seizures in the Dravet syndrome. N Engl J Med. 2017;376:2011-20.

13. Thiele E, Marsh E, French J, et al. Cannabidiol in patients with seizures associated with Lennox-Gastaut syndrome (GWPCARE4): a randomised, double-blind, placebo-controlled phase 3 trial. Lancet. 2018;391(10125):1085-96.

14. Devinsky O, Patel A, Cross JH, et al. Effect of cannabidiol on drop seizures in the Lennox-Gastaut syndrome. N Engl J Med. 2018;378:1888-97.

15. Simona L, Francesco B, Eugen T, et al. Efficacy and safety of cannabidiol in epilepsy: a systematic review and meta-analysis. Drugs. 2018;78: 1791-804.

16. Szaflarski JP, Benin EM, Cutter G, et al. Cannabidiol improves frequency and severity of seizures and reduces adverse events in an open-label add-on prospective study. Epilepsy Behav. 2018;87:131-6.

17. Rosenberg EC, Louik J, Conway E, Devinsky O, Friedman D. Quality of life in childhood epilepsy in pediatric patients enrolled in a prospective, openlabel clinical study with cannabidiol. Epilepsia. 2017;58(8):e96-100.

18. Porter B, Jacobson C. Report of a parent survey of cannabidiol-enriched cannabis use in pediatric treatment-resistant epilepsy. Epilepsy Behav. 2013;29(3):574-7. 